\title{
2.5D Face Landmarking via Scale-invariant Feature Extraction and Centroid Localization
}

\author{
SukTing Pui ${ }^{1}$, Terrin Lim and Jacey-Lynn Minoi ${ }^{2}$ \\ Faculty of Computer Science and Information Technology \\ Universiti Malaysia Sarawak \\ 94300 Kota Samarahan \\ Sarawak, Malaysia \\ ${ }^{1}$ polly_sukting@hotmail.com, ${ }^{2}$ jaceylynnminoi@gmail.com
}

\begin{abstract}
In this paper, we present and discuss our proposed method on landmarking on 2.5-dimensional (2.5D) face range images. Face landmarking plays an important role as an intermediary component in several face processing operation applications. Locating facial landmarks automatically remains a challenge. Detecting and localizing landmarks from raw face data are often performed manually by trained and experienced scientists or clinicians, and the process is usually lengthy, laborious and tedious. In order to overcome these challenges, we introduce a method that employs geometric approach, through utilizing the mean and Gaussian curvatures, primitive surfaces information to identify and label features as anatomical landmarks. In addition, comparative experiments on both automatic landmarking and manual landmarking were also performed and the results have demonstrated that the proposed method outperforms the manual landmarking in terms of obtaining distinct facial landmarks correctly and accurately.
\end{abstract}

Keywords - feature extraction; centroid localization; geometric approach; mean and Gaussian curvature

\section{INTRODUCTION}

Facial landmarking is the primary step in a number of computer vision systems including facial analysis, face recognition, facial pose estimation, medical diagnostics and multimedia applications. Facial landmarks can be classified into two groups, distinct (the eye corners, the mouth corners, the nose tip etc.) and non-distinct (wrinkles, cheek contours etc.) features [1]. As human vision system can perceive these features without any difficulties, a computer is unable to do such task as easily and effortlessly [23]. The human vision and brain mechanisms that are responsible for the detection of facial features are so complex despite the work of mathematician and computer scientists, it is still not possible to replicate facial detection accurately.

Identifying anatomical face landmarks such as the corners of the eyes (inner and outer), the nose tip, the corners of the mouth, chin, etc. is much easier to those who are familiar with the anatomy of the face. These facial landmarks contain unique structural and/or textural properties that distinguish them from their surrounding regions [10]. Therefore, these characteristics guide human beings for the search of the anatomy facial features. However, extracting these distinct landmarks in computer vision is tedious and difficult where the most important factor is the precision, where many problems remain to be solved. It is challenging as the facial features contain a large degree of face variability, facial expressions, background noises, illumination, occlusion, head pose, orientation, cluttered background etc. The detailed information is crucial and is very important for a number of clinical applications such as surgical repair to improve the facial appearance from cleft deformity [6, 7], or other orthognathic surgeries [8], in which errors in landmarking can cause serious problems.

In this paper, we propose a scale-invariant feature extraction and binarization methods to perform face landmarking on 2.5D faces. We focus attention on the distinct features as these features are consistent due to pose changes. Our objective is to first obtain distinct features on a face and label them automatically by placing landmarks. We apply Gaussian smoothing in order to extract weighted regions based on specific threshold values. Scale-space is constructed by taking the Difference of Gaussian (DoG) of images at different scales. Subsequently, within the scale-space, the weighted average of the curvature values of the elements is computed to estimate the centres or centroids. To extract feature descriptors, we create Gaussian scale-space by using mean $(\mathrm{H})$ and Gaussian (K) curvature principals. We employ the local and global descriptors of range image to identify distinct features. These feature descriptors are local regions instead of localize at one pixel in size point. Once these features are extracted, Pyramid methods are applied to acquire the filtered regions. With the aim to estimate and mark centroids on these regions, we use binarization thresholding for the centroid calculation. Lastly, we localize key information to pixel points and curves on the extracted facial features after binarization approach. We evaluate the accuracy of our method versus manual landmarking by experts. 